\title{
Japanese Herbal Kampo Hochu-Ekki-To or Juzen-Taiho-To after Surgery for Hip Fracture Does Not Reduce Infectious Complications
}

\author{
Yusuke Sasabuchi $\mathbb{D}^{1},{ }^{1,2}$ Hiroki Matsui, ${ }^{2}$ Alan Kawarai Lefor, ${ }^{3}$ Taisuke Jo, ${ }^{4}$ \\ Nobuaki Michihata, ${ }^{4}$ Kiyohide Fushimi $\mathbb{D}^{5},{ }^{5}$ and Hideo Yasunaga ${ }^{(D)}{ }^{2}$ \\ ${ }^{1}$ Data Science Center, Jichi Medical University, Tochigi, Japan \\ ${ }^{2}$ Department of Clinical Epidemiology and Health Economics, The University of Tokyo, Tokyo, Japan \\ ${ }^{3}$ Department of Surgery, Jichi Medical University, Tochigi, Japan \\ ${ }^{4}$ Department of Health Services Research, Graduate School of Medicine, The University of Tokyo, Tokyo, Japan \\ ${ }^{5}$ Department of Health Policy and Informatics, Tokyo Medical and Dental University, Graduate School of Medicine, Tokyo, Japan
}

Correspondence should be addressed to Yusuke Sasabuchi; sasabuchi-tky@umin.ac.jp

Received 14 February 2018; Revised 8 April 2018; Accepted 12 April 2018; Published 29 April 2018

Academic Editor: Kenji Watanabe

Copyright ( 2018 Yusuke Sasabuchi et al. This is an open access article distributed under the Creative Commons Attribution License, which permits unrestricted use, distribution, and reproduction in any medium, provided the original work is properly cited.

\begin{abstract}
Background. Infectious complications after hip fracture surgery are common in the elderly. Although experimental studies have suggested that kampo medicine, Hochu-ekki-to and Juzen-taiho-to, can prevent infectious complications, only a few small clinical studies have been published to date. Primary Study Objective. The aim of the present study is to investigate the impact of Hochuekki-to or Juzen-taiho-to on postoperative infectious complications in patients undergoing surgery for hip fracture. Methods and Design. In this retrospective cohort study using a nationwide inpatient database in Japan, we performed propensity score matching to compare patients who did or did not receive kampo medicine after surgery for hip fracture. Settings. A nationwide inpatient database. Participants. Patients who did or did not receive kampo medicine after surgery for hip fracture. Intervention. Kampo medicine after surgery for hip fracture. Primary Outcome Measures. Infectious complications. Results. The proportions of postoperative infectious complications were not significantly different between the 424 propensity-matched pairs with and without kampo medicine (11 versus $8, P=0.644$ ). Conclusion. The present study suggests that Hochu-ekki-to or Juzen-taiho-to postoperatively is not associated with decreased occurrence of infectious complications in patients who underwent surgery for hip fracture.
\end{abstract}

\section{Introduction}

Hip fractures are common in the elderly. With an aging population, the incidence of hip fractures is increasing $[1,2]$. The incidence of hip fractures worldwide is estimated to be 2.6 million by 2025 and to exceed 7 million by 2050 [3]. Pneumonia and surgical site infections are common complications after surgery for hip fracture $[4,5]$. These complications have been reported to increase mortality after surgery for hip fracture [6, 7].

Japanese herbal kampo medicines have become popular in Japan [8]. Hochu-ekki-to and Juzen-taiho-to are two major kampo medicines, which are used to treat immunocompromised individuals $[9,10]$. Experimental studies have suggested that Hochu-ekki-to and Juzen-taiho-to can prevent infectious diseases through enhancing the immune system [11-15]. Although Hochu-ekki-to and Juzen-taihoto may be promising to prevent infectious diseases, only a few small clinical studies [16-21] have been published to date.

The aim of the present study is to investigate the impact of Hochu-ekki-to or Juzen-taiho-to on postoperative infectious complications in patients undergoing surgery for hip fracture, using a nationwide inpatient database in Japan. 


\section{Materials and Methods}

The Institutional Review Board of the University of Tokyo approved this study. Informed consent was waived due to the anonymous nature of the data.

2.1. Data Source. Patient data were extracted from the Diagnosis Procedure Combination database, which is a nationwide administrative claims database with discharge abstracts representing approximately $50 \%$ of all admissions to acute care hospitals in Japan [22, 23]. The Diagnosis Procedure Combination database provides (i) patient demographic data, (ii) admission-precipitating diagnosis, preexisting comorbidities on admission, and complications during hospitalization coded with the International Classification of Diseases, Tenth Revision (ICD-10) codes, (iii) hospital identification number, (iv) dates of procedures performed and dates of medications administered, (v) discharge status, and (vi) dates of hospital or intensive care unit admission and discharge. Physicians in charge record data for diagnoses, comorbidities, and discharge status.

2.2. Patients. In the present study, patients were included if they (i) had a hip fracture (ICD-10 S720, S721, or S722) as the admission-precipitating diagnosis, (ii) underwent surgery (open reduction and internal fixation or hemiarthroplasty) for hip fracture, and (iii) were discharged between July 2010 and March 2014. Exclusion criteria included the following: (i) age < 40 years; (ii) body mass index data being unavailable, less than $10 \mathrm{~kg} / \mathrm{m}^{2}$ or more than $70 \mathrm{~kg} / \mathrm{m}^{2}$; (iii) number of hospital beds being not available.

2.3. Exposure of Interest, Outcomes, and Other Variables. The exposure of interest was whether patients received Hochuekki-to or Juzen-taiho-to within seven days of surgery. These patients were defined as the kampo group. Other patients were defined as the control group.

Assessed outcomes include infectious complications and in-hospital deaths. Infectious complications include surgical site infection (SSI, ICD-10, T793, or T814), hospital acquired pneumonia (J10-18), and sepsis (A40 or A41).

Other variables evaluated include age, gender, body mass index, comorbidities extracted using algorithms developed by Quan et al. [24], fracture location, type of surgery, days between admission and the surgery, treatment year, the volume of red cell transfusion, and anesthesia time. As an index for severity, use of mechanical ventilation, administration of catecholamines, administration of red cell transfusion, renal replacement therapy, and admission to a high intensity care unit such as an intensive care unit or high care unit within seven days of surgery are also extracted.

2.4. Statistical Analysis. Continuous variables are presented as the average with the standard deviation. Categorical variables are presented as the number with a percentage. Differences in the baseline characteristics between the kampo group and control group are compared using standardized differences. A standardized difference $<10 \%$ indicates a negligible imbalance in baseline characteristics between groups [25].

To estimate the probability that a patient would receive Hochu-ekki-to or Juzen-taiho-to medicine, a propensity score was calculated for each patient using a multivariable logistic regression for receiving kampo medicine. As independent variables, the baseline characteristics shown in Table 1 are incorporated. Patients who received kampo medicine were 1-to-1 matched with patients in the control group on the basis of nearest neighbor matching without replacement. The caliper was set at $20 \%$ of the standard deviation of the propensity score. Outcomes between the two groups were compared using Fisher's exact test. A $P$ value $<0.05$ is considered statistically significant. Propensity score matching is performed using "Matching" package of $\mathrm{R}$ version 3.1.3 (The R Foundation, Vienna, Austria). All other analyses are performed using SPSS version 22 (SPSS Inc., Chicago, Illinois, US).

\section{Results}

After inclusion and exclusion criteria were applied, a total of 201,900 patients were included in the analysis (Figure 1). Of these, 424 patients received Hochu-ekki-to (259 patients) or Juzen-taiho-to (165 patients) within seven days of surgery.

Table 1 shows a comparison of baseline characteristics of patients in the kampo group and the control group before and after propensity score matching. Before propensity score matching, patients in the kampo group were more likely to have chronic pulmonary disease, peptic ulcer, and malignancy. Anesthesia time was shorter and the amount of transfusion was less in the kampo group. After propensity score matching, baseline characteristics between the two groups are well balanced.

Table 2 shows outcomes comparing the two groups. After propensity score matching, neither infectious complications nor in-hospital death was significantly different between the groups.

\section{Discussion}

In this retrospective study using a nationwide administrative database in Japan, postoperative use of Hochu-ekki-to or Juzen-taiho-to was not associated with decreased occurrence of postoperative infectious complications or in-hospital death for patients undergoing surgery for hip fracture.

Previous animal studies have suggested immunostimulating properties of Hochu-ekki-to and Juzen-taiho-to. However, only a few small clinical studies have been conducted. Hochu-ekki-to prevented immunosuppression induced by surgery [17] or inhibited rhinovirus infection in airway epithelium [18]. Clinical studies of Juzen-taiho-to have shown improved host immunity in patients with brain tumors [21] and a significantly decreased frequency of otitis media was in children $[19,20]$.

There are several possible explanations why the results of the present study failed to show a favorable effect of the drugs. First, the timing of administration of the kampo medicine may not be appropriate. In the present study, 


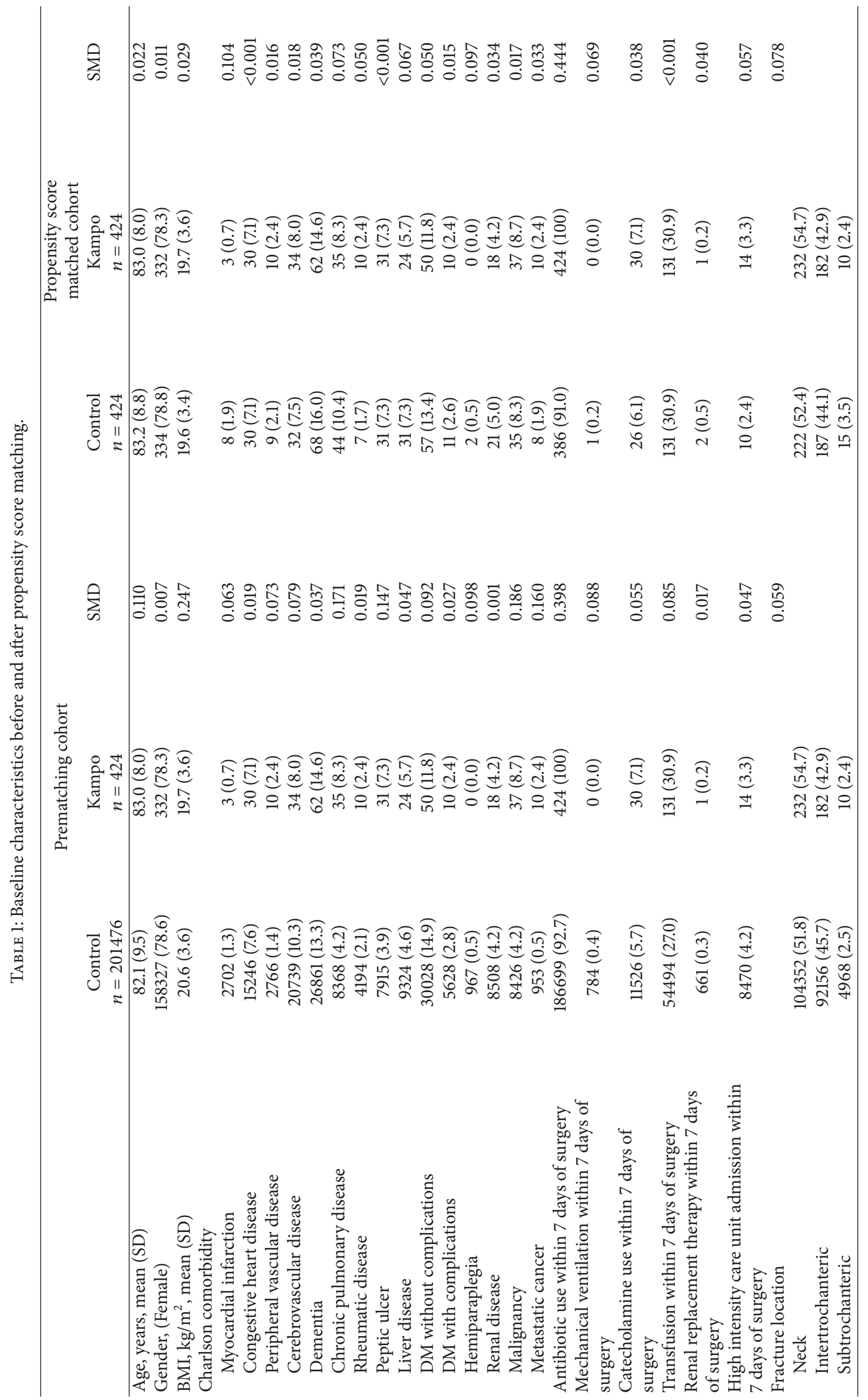




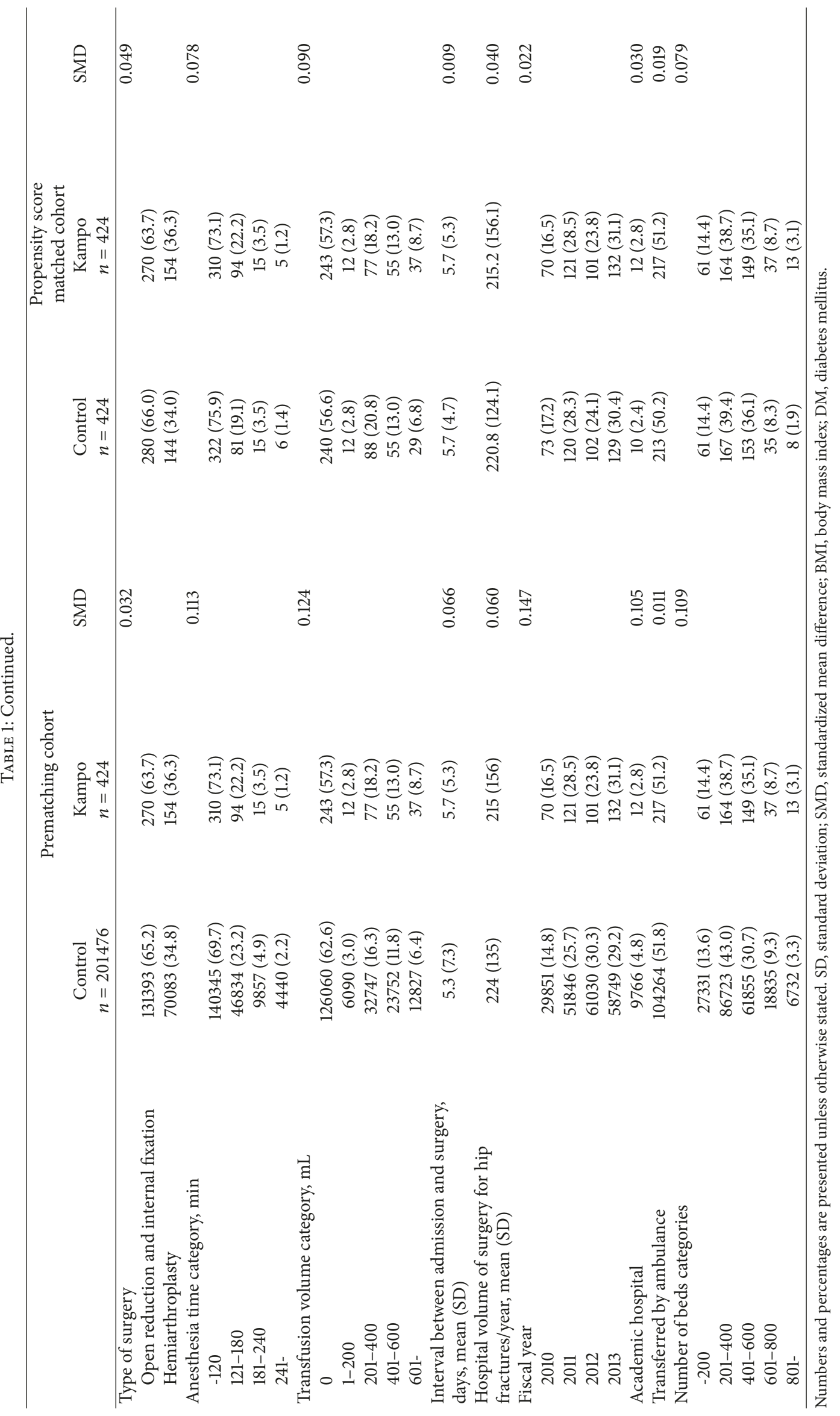




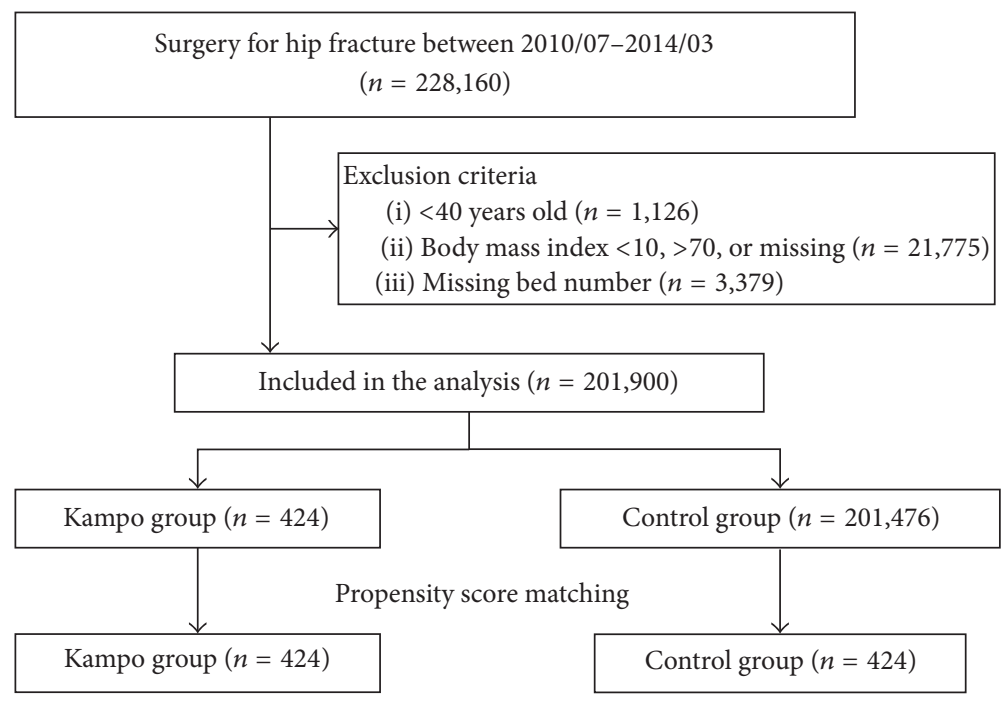

Figure 1: Study flow diagram.

TABLE 2: Outcomes before and after propensity score matching.

\begin{tabular}{|c|c|c|c|c|c|c|}
\hline & \multicolumn{3}{|c|}{ Prematching cohort } & \multicolumn{3}{|c|}{ Propensity score matched cohort } \\
\hline & $\begin{array}{c}\text { Control } \\
n=201476\end{array}$ & $\begin{array}{l}\text { Kampo } \\
n=424\end{array}$ & $P$ & $\begin{array}{l}\text { Control } \\
n=424\end{array}$ & $\begin{array}{l}\text { Kampo } \\
n=424\end{array}$ & $P$ \\
\hline In-hospital death, $n(\%)$ & $3552(1.8)$ & $8(1.9)$ & 0.852 & $6(1.4)$ & $8(1.9)$ & 0.394 \\
\hline Infectious complications, $n(\%)$ & $4647(2.3)$ & $12(2.8)$ & 0.578 & $6(1.4)$ & $12(2.8)$ & 0.234 \\
\hline Hospital-acquired pneumonia, $n(\%)$ & $2830(1.4)$ & $6(1.4)$ & & $5(1.2)$ & $6(1.4)$ & \\
\hline Surgical site infection, $n(\%)$ & $1239(0.6)$ & $3(0.7)$ & & $1(0.2)$ & $3(0.7)$ & \\
\hline Sepsis, $n(\%)$ & $710(0.4)$ & $4(0.9)$ & & $2(0.5)$ & $4(0.9)$ & \\
\hline
\end{tabular}

the kampo group received Hochu-ekki-to or Juzen-taihoto within seven days of surgery, whereas patients received kampo medicine before surgery in a previous study [17]. Postoperative administration of kampo medicine may not sufficiently reduce postoperative infectious complications in patients undergoing surgery for hip fracture. Second, previous studies mainly included infection-prone patients. Although the patients in the present study were older (mean age more than 80 years), most of these patients may have been immunocompetent. Hochu-ekki-to or Juzen-taiho-to may be effective in immunocompromised patients. Lastly, the lack of a significant reduction in infective complications may be due to type II error. Further study, including larger numbers of patients, is warranted.

We acknowledge that the present study has several limitations. First, despite the use of propensity score matching, residual confounding may bias the results because of the retrospective nature of the study. Second, the study does not show a preventive effect for infectious complications of kampo medicine; however, the sample size may not be sufficiently large to detect a difference between the groups. Third, the database does not contain information before admission or after discharge. Patients may have had Hochuekki-to or Juzen-taiho-to before surgery or may have suffered from infectious complications after discharge. Fourth, patients who received Hochu-ekki-to or Juzen-taiho-to were from 238 of 1147 hospitals. The fact that patients who received these kampo medicines were from a relatively small fraction of hospitals may bias the results. Fifth, antibiotic usage was not accounted for in estimating the propensity score because it was difficult to differentiate antibiotics use for prevention from use for treatment.

In conclusion, postoperative Hochu-ekki-to or Juzentaiho-to is not associated with a decrease in the occurrence of infectious complications after surgery for hip fracture. It is possible that receiving Hochu-ekki-to or Juzen-taiho-to only after surgery is not sufficient to prevent postoperative complications.

\section{Data Availability}

Data cannot be made publicly available for ethical reasons as the data are patient data. The data are available to interested researchers upon request to the corresponding author, pending ethical approval.

\section{Disclosure}

The funding sources had no role in study design; in the collection, analysis, and interpretation of data; in the writing of the report; and in the decision to submit the article for 
publication. This study was performed at the University of Tokyo.

\section{Conflicts of Interest}

None of the authors has any potential financial conflicts of interest related to this manuscript.

\section{Acknowledgments}

This work was supported by grants from the Ministry of Health, Labour and Welfare, Japan; Ministry of Education, Culture, Sports, Science and Technology, Japan; and the Japan Agency for Medical Research and Development (AMED). The Department of Health Services Research, Graduate School of Medicine, the University of Tokyo, is funded in part by Tsumura Pharmaceuticals Co. Ltd. (Tokyo, Japan).

\section{References}

[1] M. Parker and A. Johansen, "Hip fracture," British Medical Journal, vol. 333, no. 7557, pp. 27-30, 2006.

[2] H. Orimo, Y. Yaegashi, T. Hosoi et al., "Hip fracture incidence in Japan: estimates of new patients in 2012 and 25-year trends," Osteoporosis International, vol. 27, no. 5, pp. 1777-1784, 2016.

[3] B. Gullberg, O. Johnell, and J. A. Kanis, "World-wide projections for hip fracture," Osteoporosis International, vol. 7, no. 5, pp. 407413, 1997.

[4] E. C. Folbert, J. H. Hegeman, R. Gierveld et al., "Complications during hospitalization and risk factors in elderly patients with hip fracture following integrated orthogeriatric treatment," Archives of Orthopaedic and Trauma Surgery, vol. 137, no. 4, pp. 507-515, 2017.

[5] A. M. Ali and C. E. R. Gibbons, "Predictors of 30-day hospital readmission after hip fracture: a systematic review," Injury, vol. 48, no. 2, pp. 243-252, 2017.

[6] H. Q. Sheikh, F. S. Hossain, A. Aqil, B. Akinbamijo, V. Mushtaq, and H. Kapoor, "A comprehensive analysis of the causes and predictors of 30-day mortality following hip fracture surgery," CiOS Clinics in Orthopedic Surgery, vol. 9, no. 1, pp. 10-18, 2017.

[7] S. Starčević, V. Šuljagić, D. Stamenković, D. Bokonjić, and S. Munitlak, "In-hospital mortality analysis in patients with proximal femoral fracture operatively treated by hip arthroplasty procedure," Vojnosanitetski Pregled, vol. 73, no. 3, pp. 251-255, 2016.

[8] L. Teng, Q. Zu, G. Li et al., "Herbal medicines: challenges in the modern world. Part 3. China and Japan," Expert Review of Clinical Pharmacology, vol. 9, no. 9, pp. 1225-1233, 2016.

[9] S. Nishida, "Effect of Hochu-ekki-to on asymptomatic MRSA bacteriuria," Journal of Infection and Chemotherapy, vol. 9, no. 1, pp. 58-61, 2003.

[10] I. Saiki, K. Koizumi, H. Goto et al., "The long-term effects of a Kampo medicine, juzentaihoto, on maintenance of antibody titer in elderly people after influenza vaccination," EvidenceBased Complementary and Alternative Medicine, vol. 2013, Article ID 568074, 8 pages, 2013.

[11] Y. Yamaoka, T. Kawakita, and K. Nomoto, "Protective effect of a traditional Japanese medicine Hochu-ekki-to (Chinese name: Bu-zhong-yi-qi-tang), on the susceptibility against Listeria monocytogenes in infant mice," International Immunopharmacology, vol. 1, no. 9-10, pp. 1669-1677, 2001.
[12] S. Abe, S. Tansho, H. Ishibashi, G. Akagawa, Y. Komatsu, and H. Yamaguchi, "Protection of immunosuppressed mice from lethal Candida infection by oral administration of a Kampo medicine, Hochu-ekki-to," Immunopharmacology and Immunotoxicology, vol. 21, no. 2, pp. 331-342, 1999.

[13] K. Mori, T. Kido, H. Daikuhara et al., "Effect of Hochu-ekkito (TJ-41), a Japanese herbal medicine, on the survival of mice infected with influenza virus," Antiviral Research, vol. 44, no. 2, pp. 103-111, 1999.

[14] S. Abe, S. Tansho, H. Ishibashi, N. Inagaki, Y. Komatsu, and H. Yamaguchi, "Protective effect of oral administration of a traditional medicine, Juzen-taiho-to, and its components on lethal Candida albicans infection in immunosuppressed mice," Immunopharmacology and Immunotoxicology, vol. 20, no. 3, pp. 421-431, 1998.

[15] T. Matsumoto, M. H. Sakurai, H. Kiyohara, and H. Yamada, "Orally administered decoction of Kampo (Japanese herbal) medicine, 'Juzen-Taiho-To' modulates cytokine secretion and induces NKT cells in mouse liver," International Journal of immunopharmacology, vol. 46, no. 2, pp. 149-161, 2000.

[16] K. Hamazaki, S. Sawazaki, M. Itomura et al., "No effect of a traditional Chinese medicine, Hochu-ekki-to, on antibody titer after influenza vaccination in man: a randomized, placebocontrolled, double-blind trial," Phytomedicine, vol. 14, no. 1, pp. 11-14, 2007.

[17] M. Kimura, T. Sasada, M. Kanai et al., "Preventive effect of a traditional herbal medicine, Hochu-ekki-to, on immunosuppression induced by surgical stress," Surgery Today, vol. 38, no. 4, pp. 316-322, 2008.

[18] M. Yamaya, T. Sasaki, H. Yasuda et al., "Hochu-ekki-to inhibits rhinovirus infection in human tracheal epithelial cells," British Journal of Pharmacology, vol. 150, no. 6, pp. 702-710, 2007.

[19] Y. Maruyama, S. Hoshida, M. Furukawa, and M. Ito, "Effects of Japanese herbal medicine, Juzen-taiho-to, in otitis-prone children-a preliminary study," Acta Oto-Laryngologica, vol. 129, no. 1, pp. 14-18, 2009.

[20] M. Ito, Y. Maruyama, K. Kitamura et al., "Randomized controlled trial of juzen-taiho-to in children with recurrent acute otitis media," Auris Nasus Larynx, vol. 44, no. 4, pp. 390-397, 2017.

[21] M. Miyagami and Y. Katayama, "Improvement of hostimmunity by adjuvant therapy with Juzen-Taiho-To for patients with brain tumors," Journal of Neurological Surgery, vol. 31, no. 4, pp. 401-409, 2003.

[22] S. Matsuda, K. Fujimori, K. Kuwabara, K. B. Ishikawa, and K. Fushimi, "Diagnosis procedure combination as an infrastructure for the clinical study," Asian Pacific Journal of Disease Management, vol. 5, no. 4, pp. 81-87, 2011.

[23] H. Yamana, H. Matsui, Y. Sasabuchi, K. Fushimi, and H. Yasunaga, "Categorized diagnoses and procedure records in an administrative database improved mortality prediction," Journal of Clinical Epidemiology, vol. 68, no. 9, pp. 1028-1035, 2015.

[24] H. Quan, B. Li, C. M. Couris et al., "Updating and validating the charlson comorbidity index and score for risk adjustment in hospital discharge abstracts using data from 6 countries," American Journal of Epidemiology, vol. 173, no. 6, pp. 676-682, 2011.

[25] P. C. Austin, "An introduction to propensity score methods for reducing the effects of confounding in observational studies," Multivariate Behavioral Research, vol. 46, no. 3, pp. 399-424, 2011. 


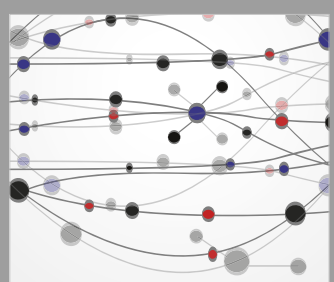

The Scientific World Journal
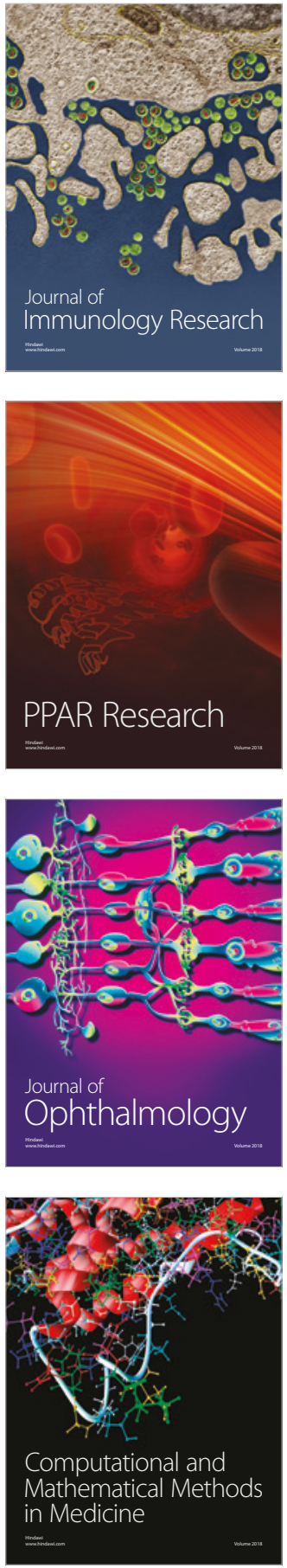

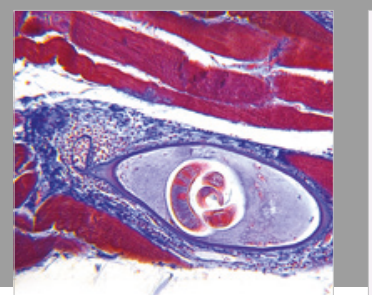

Gastroenterology Research and Practice

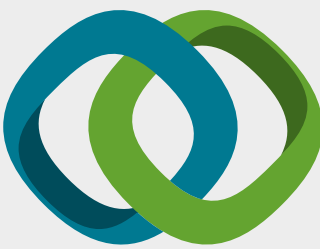

\section{Hindawi}

Submit your manuscripts at

www.hindawi.com
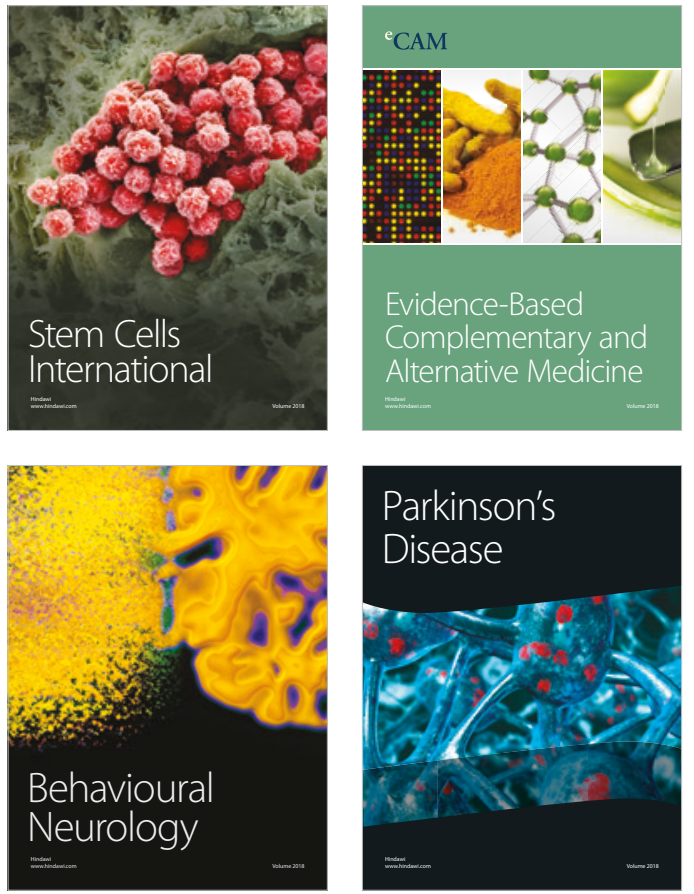

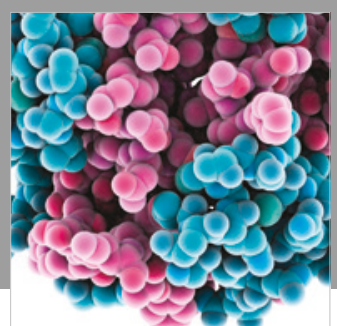

ournal of

Diabetes Research

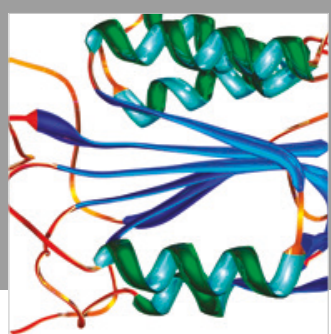

Disease Markers
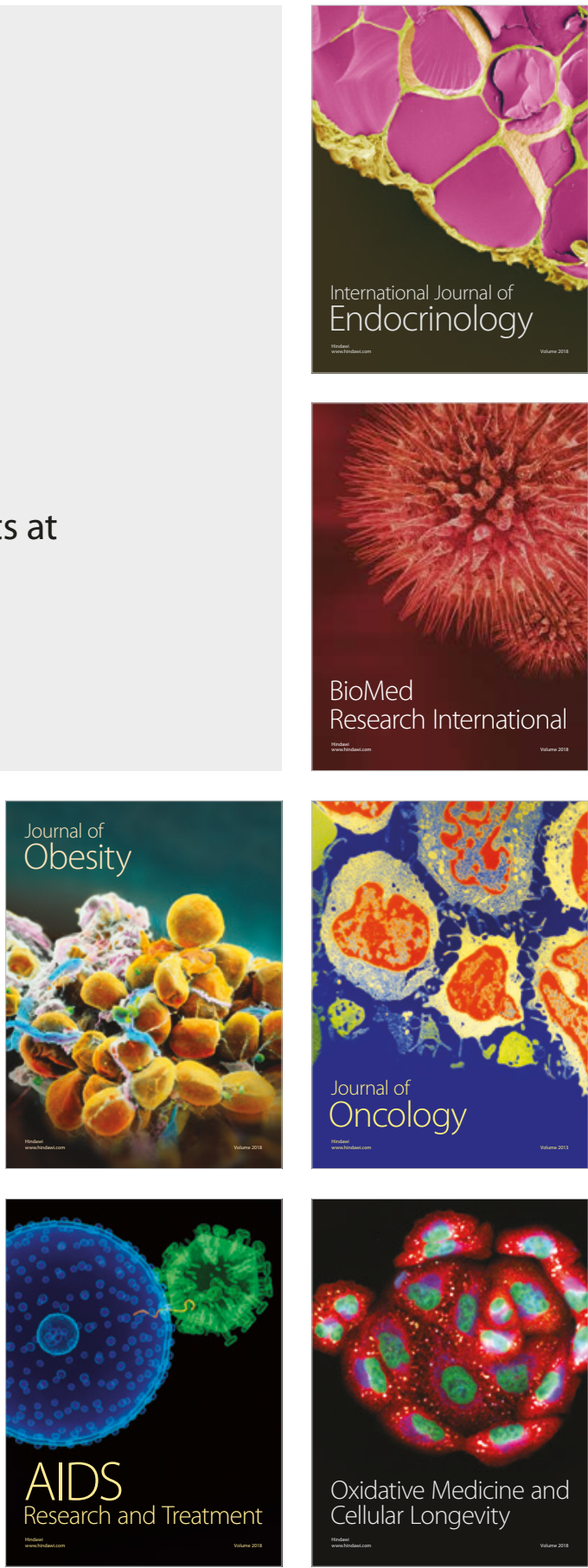